ENCYCLOPEDEE Encyclopédie berbère

BERBERE

21 | 1999

21 | Gland - Hadjarien

\title{
Guelaya ou Qelaya
}

Qal'iya ou Kal'iya

\section{H. Al Figuigui}

Traducteur : J. Vignet-Zunz

\section{OpenEdition}

\section{Journals}

Édition électronique

URL : http://journals.openedition.org/encyclopedieberbere/1805

DOI : 10.4000/encyclopedieberbere.1805

ISSN : 2262-7197

\section{Éditeur}

Peeters Publishers

\section{Édition imprimée}

Date de publication : 1 septembre 1999

Pagination : 3224-3229

ISBN : 2-7449-0097-4

ISSN : 1015-7344

\section{Référence électronique}

H. Al Figuigui, « Guelaya ou Qelaya », Encyclopédie berbère [En ligne], 21 | 1999, document G78, mis en ligne le 01 juin 2011, consulté le 24 septembre 2020. URL : http://journals.openedition.org/ encyclopedieberbere/1805; DOI : https://doi.org/10.4000/encyclopedieberbere.1805

Ce document a été généré automatiquement le 24 septembre 2020

(C) Tous droits réservés 


\section{Guelaya ou Qelaya}

Qal'iya ou Kal'iya

\section{H. Al Figuigui}

Traduction : J. Vignet-Zunz

1 Tribu marocaine appartenant géographiquement au Rif Oriental, occupant l'angle nord-est de l'actuelle province de Nador qui regroupe les villes de Melilla/Malîla (sous l'autorité de l'Espagne), Nador, capitale de la province, Azghanghan et Salwân. Le territoire occupe une superficie de $984 \mathrm{~km}^{2}$. La tribu apparaît aujourd'hui dans le régime administratif local sous la dénomination Cercle de Guelaya.

\section{Le nom de Guelaya}

2 Ce nom dérive du mot arabe al-qalâ', pluriel d'al-qal'at. L'histoire médiévale permet d'associer ce nom à Qal'at Kert, ou Qal'at Gârat, la plus fameuse fortification militaire des Guelaya, antérieure à l'expansion de l'islam au Maroc.

3 À partir du début du VII ${ }^{\mathrm{e}} \mathrm{H}$./ XIII siècle, la tribu se nomma "Territoire des Forts ", balad al -qulû', s'étendant du plateau de Tâzûdâ (900 m) aux crêtes (Sîdî Ahmad al-Hâj, Wisân et Tîdhînt) qui marquent le sommet volcanique du Mont des Guelaya, que les Espagnols dénomment Gurugu, c'est-à-dire le groupe de crêtes qui se voient depuis l'entrée occidentale de la tribu jusqu'à l'Oued Kert. Le nom de Guelaya apparut finalement avec l'installation des Mérinides à Qal'at Tâzûdâ et leur conquête du Rif Oriental, en 610 H./ 1213. 


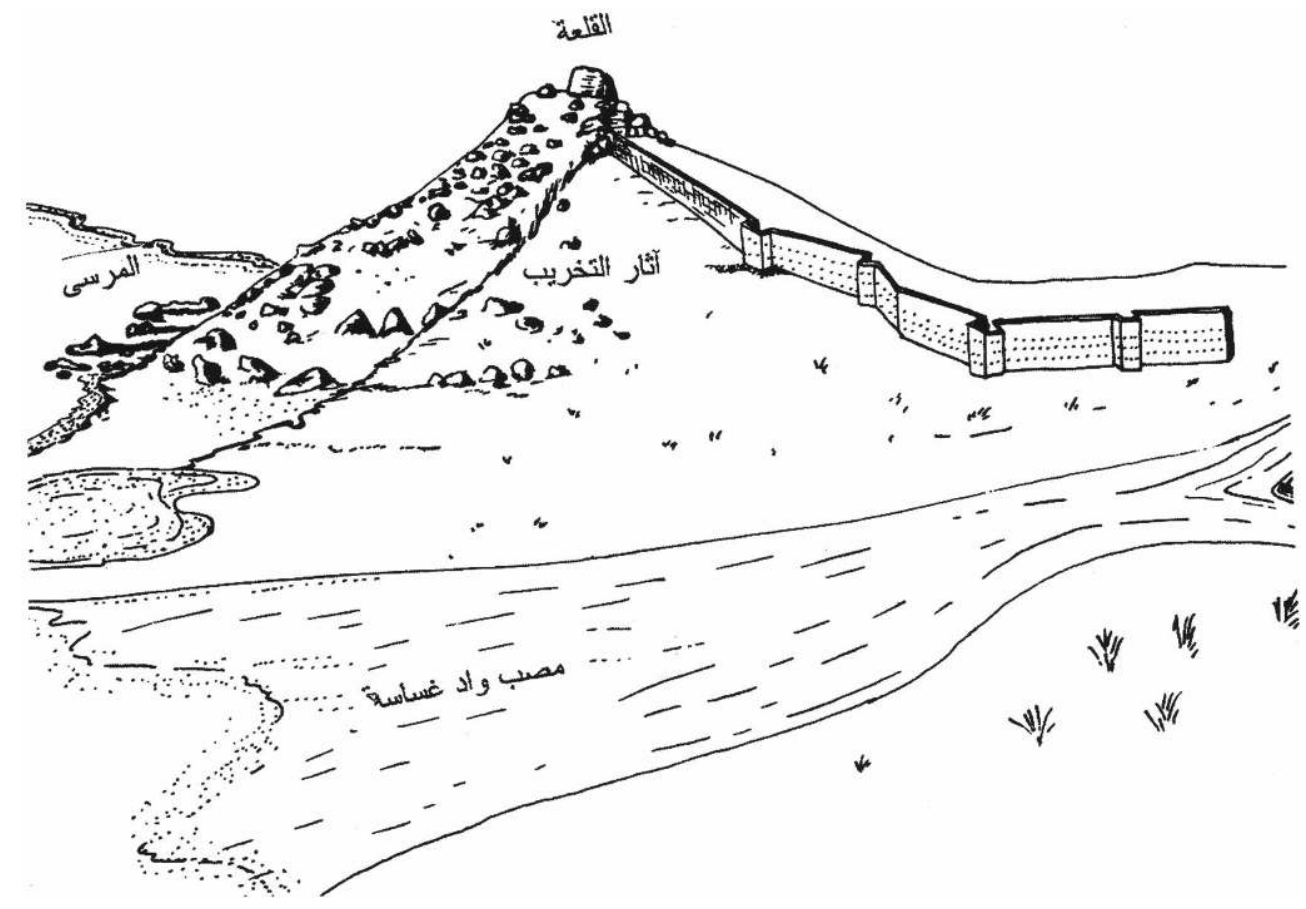

\section{Structure sociale}

4 En réalité, ce que l'on a coutume d'appeler «tribu de Guelaya » est la réunion de familles, ou groupes, originaires de diverses régions. Néanmoins, il y avait, dans les premières années de l'ère musulmane au Maroc, d'autres groupes originaires, eux, de l'antique Guelaya. Ainsi les Bani Ouartada, de filiation Batuya, seigneurs du territoire jusqu'au $\mathrm{V}^{\mathrm{e}} \mathrm{H} . / \mathrm{XI}^{\mathrm{e}}$ siècle. Leurs voisins étaient l'ensemble des Ghasâsa, d'origine Nafza*. Auxquels il faut ajouter des Matmata* et des Bani Yafran.

5 Le territoire Guelaya fut envahi dès le début du VII ${ }^{\mathrm{e}} \mathrm{H} . / \mathrm{XIII}^{\mathrm{e}}$ siècle par un grand nombre de familles Zanata dirigées par l'armée des Bani Marin et leurs alliés arabes, Banu Hilal et Bani M'aqil, arrivant du sud-est du Maroc et formant le gros de l'immigration. S'infiltrèrent également d'autres familles originaires de Tlemcen, Oran, Bani Iznassen, Ghiata et des tribus du Rif Oriental. De ces bouleversements procèdent les fractions qu'on peut encore noter de nos jours.

6 L'une des conséquences les plus marquantes de ces transformations est la nouvelle structure sociale qui est apparue entre les VII et x H./XIII et $\mathrm{XVI}^{\mathrm{e}}$ siècles. Al-Hasan alWazzan, Jean-Léon l'Africain, ne nous en présente pas un tableau précis. Un autre auteur, anonyme, nous livre en 939 H./1533 un ensemble détaillé de la structure qui ne se différencie en rien de l'actuelle. Grâce à sa relation, nous savons que la tribu se composait de cinq parties, ou fractions, chacune relevant de la notion de tribu, qui se dénommaient al-khumus, le quint, suivi du nom du groupe. 


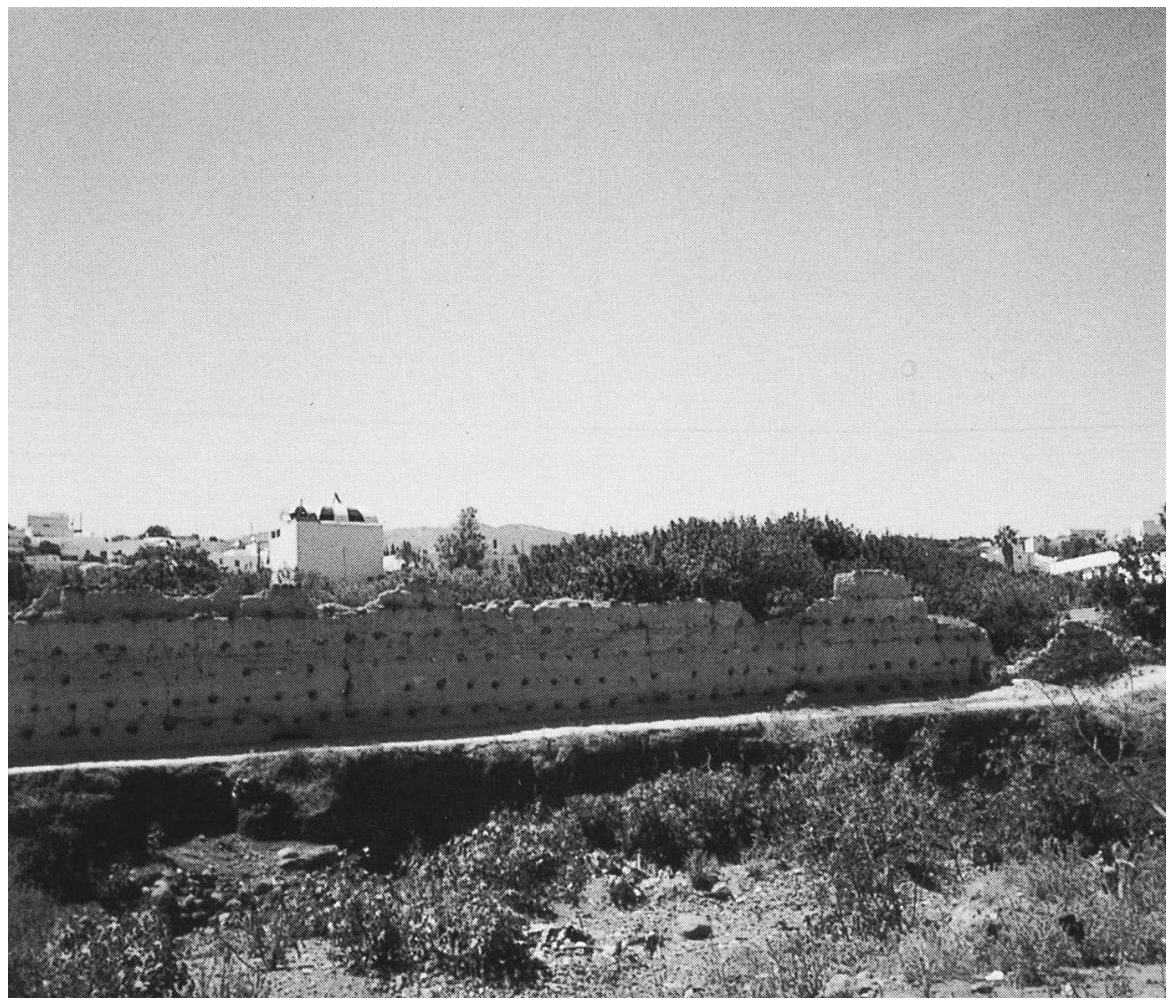

7 Les voici :

- Bani Chicar : ils comprenaient au X H./Xvi ${ }^{\mathrm{e}}$ siècle vingt douars occupant la presqu'île de Hork (Cap Tres Forcas ou des Trois Fourches); ils sont aujourd'hui quarante-six, regroupés en quatre fractions. Sur son territoire se trouvent des ruines de Qal'at Tâzûdâ, sur le plateau du même nom.

- Bani Bûgâfar : ils comprennent aujourd'hui quatorze douars répartis en trois fractions qui occupent la zone nord-ouest des Guelaya, entre la Méditerranée et l'Oued Kert. Les ruines de la vieille cité de Ghasâsa sont sur son rivage.

- Bani Sîdâl : ancien nom, Al-Ga'da; vingt-et-un douars au Xvi siècle sur la façade occidentale du Mont des Guelaya, s'étendant jusqu'à la rive droite de l'Oued Kert; quarante-neuf aujourd'hui, répartis en cinq fractions.

- Mazzûja : de vingt-et-un douars, sur le versant est du Mont des Guelaya et une partie du littoral de la lagune de Bû'arg, on passe à soixante-dix-huit douars, répartis en neuf fractions.

- Bani Bûyafrûr : leurs douars se déploient autour du sommet du Jabal Wisân jusqu'à la limite qui sépare le poste militaire de Tînîmârt de l'Oued Salwân. On y trouve la Qaçba de Salwân, fondée sur ordre de Moulay Isma'îl à la fin du XI ${ }^{\mathrm{e}} \mathrm{H} . / \mathrm{XVII}$ e siècle.

8 Cette organisation de tribus en " cinq cinquièmes » est très répandue chez les Berbères du Maroc. Elle était connue dès l'Antiquité sous l'Empire romain (cf. E.B. C67. Cinq, t. XIII, p. 1958-1960).

\section{Histoire}

9 La première référence historique à la région est le comptoir punico-carthaginois de RS ADR (Rusadir). Toute l'histoire antique du territoire se rapporte à cette implantation et 
à celle de leurs successeurs romains et byzantins. Cela nous permet d'évoquer, bien que de manière confuse, ces premiers habitants de Guelaya.

10 Au cours des années qui suivent la conquête musulmane, deux faits importants se détachent : l'apparition du nom de Malîla, à la place de RS ADR ; et l'existence de Qal'at Kert, fort de Bani Wartada (la Qal'at Jârat d'Al-Bakri).

11 Ces deux faits ont une signification primordiale, ils signalent le rôle d'intermédiaires avec Al-Andalus dans le commerce de l'or africain auquel ces deux places se sont hissées.

Dans les premières années du IV $\mathrm{H}$./ $\mathrm{X}^{\mathrm{e}}$ siècle, il ne fut pas possible à Guelaya de rester à l'écart de la guerre qui éclata entre les Bani Marwân d'Al-Andalus et les Fatimides de Tunis, puisque Malîla et Qal'at Kert tombaient en 314 h./926 aux mains des envahisseurs venus de l'Ifriqiya.

13 En 459 H./1066, Guelaya fut soumise à l'autorité de Muhammad ibn Idris Al-Hammûdî, prince détrôné de Malaga et Ceuta, réfugié à Alméria d'où les Guelaya firent appel à lui. Le règne de cet émir durera jusqu'à l'arrivée des Almoravides à Guelaya en 473 H./1080. En 536 H./l 141, les Almohades s'emparent de la ville de Malîla.

L'histoire de Guelaya se fait plus précise sous les Mérinides, quand ceux-ci s'établissent à Tâzûda, en 610 H./1213, ce qui fut la cause des transformations sociales énoncées plus haut. Il convient d'ajouter le développement de la ville de Ghasâsa, qui fut renommée sous les Mérinides.

\section{Ghasâsa}

Le mot provient très probablement de la racine tamazight akhsas (ahsas), la tête humaine, avec ekhsasen comme pluriel. On sait qu'il s'applique à l'un des groupes les plus anciennement établis sur le territoire des Guelaya, appartenant au grand ensemble berbère des Nafza.

L'agglomération de Ghasâsa occupait, durant les premiers siècles de l'Islam, le littoral oriental de la presqu'île de Hork et jouait un rôle important dans le commerce de l'or du Soudan comme relais vers les ports d'Al-Andalus. Aujourd'hui, ce nom n'est plus porté que par les ruines de la vieille cité, sur le territoire de la fraction de Bani Bûgâfar. Ce sont les Bani Marin qui fondèrent la cité de Ghasâsa et sa forteresse quand ils s'installèrent dans la région en 610/1213. Elle est distante de quelque dix-huit km de Malîla, sur une colline littorale située à la limite des fractions/tribus de Bani Chicar et Bani Bûgâfar.

Ils bâtirent une forteresse dominant directement la mer, sur le sommet aplani de la colline, à une altitude de soixante dix-sept mètres. Elle portait le nom de Al-Qulla, dérivé de Al-Qala'. On la connaissait aussi sous le nom de Kudiat Ghasâsa, la colline de Ghasâsa. Cette forteresse est aujourd'hui totalement détruite.

La cité proprement dite occupait le versant sud de la colline, entourée d'une enceinte de deux kilomètres. Elle disposait d'un port de moyenne profondeur, sans aucun abri naturel, qui fut néanmoins le port le plus renommé et le plus florissant des Bani Martin dans le Rif oriental, pour leurs rapports avec Al-Andalus. Il était également fréquenté par les marchands européens, particulièrement les Vénitiens et les Aragonais. Il apparaît dans les sources médiévales sous le nom de Alcudia Albaida, la colline blanche. 

Castillans, en 903 H./1497. Toute la période qui va de cette date jusqu'à la fin du XIX siècle se caractérise par des mouvements de résistance continus contre la présence militaire des Espagnols à Malîla. On retiendra surtout le siège de la ville par l'armée marocaine en la présence du sultan Muhammad Ben Abdallah, siège qui dura du 9 décembre 1774 au 16 mars 1775.

\section{Ham} mieux cette période est l'héroïque mouvement du mujahid Al-Charif Muhammad Amazian Al-Gal'i dirigé contre l'invasion espagnole du territoire des Guelaya pendant les années 1909-1912 et qui prit fin avec l'extension de la domination ibérique sur ce territoire et l'établissement du Protectorat espagnol sur le Nord du Maroc.

\section{BIBLIOGRAPHIE}

AL-FIGUIGUI Hassan, Al-muqâwama al-maghribiya lil wûjûd al-isbânî bi Malîlah, doctorat de $3^{\mathrm{e}}$ cycle, Rabat, 1997.

AL-FIGuigui Hassan, « Malîlah hâdira Qulu' Kart », Dar Niaba, Études d'Histoire Marocaine, 7-9, 1985-86.

AL-FIGUIGUi Hassan, Sîdî Muhammad Bin 'Abdallah wa qadiyat Malîla al-muhtala, Imprimerie Royale, Rabat, 1996.

JEAN-LÉON L'AFRICAIN, (Al-Hasan Al-Wazzan), Description de l'Afrique. Anonyme, Nasab kabilat qal'iya, ms, Bibliothèque Générale, Rabat.

BERNARDES A., Historia de los Reyes Catolicos.

Comision historica de las campañas de Marruecos, Geografica de Marruecos, protectorados y

posesiones de España en Africa, t. 2, Madrid, 1936.

Encyclopédie berbère, 21 | 1999 
Comision historica de las campañas de Marruecos, Zâbt al-umûr al-wataniya fí al-man-taqa alkhalifia, Tétouan, 1951.

DUFFOURQ C, L'Espagne catalane et le Maghreb aux XIII et XIV siècles, Paris, 1966.

FERNANDEZ DE CASTRO Y PEDRERA R., El Rif, Los territorios de Guilaia y Quebdana, Malaga, 1911.

FERNANDEZ DE CASTRO Y PEDRERA R., Historia y exploracion de la ruinas de Cazaza, Madrid, 1943.

FERNANDEZ DE CASTRO Y PEDRERA R., Melilla Prehispanica, Madrid, 1945. Ibn Khaldun, Histoire, 1.1 (p. 171, 227) et t. IV (p. 416).

IBN ABI ZAR, Al-Kertass, Passim.

LOURIDO DIAZ R., « Estrategia militar y diplomatica previa al asidio de Melilla », Revista Historica Militar, 36, 1974.

MARMOL Carbajal, L'Afrique.

DE MEDINA E, Cronica del Conde de Medina Sidonia.

S.I.H.M., Espagne, t. 1.

A.G.S., Estado L. 447.

ZURITA J., Anales de la Corona de Aragon, Zaragoza, 1670.

ZURITA J., Historia del Rey D. Hernando.

INDEX

Mots-clés : Maroc, Tribu 